\title{
Mathematical problem-solving in scientific practice
}

\author{
Davide Rizza ${ }^{1}$
}

Received: 9 March 2021 / Accepted: 28 August 2021 / Published online: 23 September 2021

(c) The Author(s) 2021

\begin{abstract}
In this paper I study the activity of mathematical problem-solving in scientific practice, focussing on enquiries in mathematical social science. I identify three salient phases of mathematical problem-solving and adopt them as a reference frame to investigate aspects of applications that have not yet received extensive attention in the philosophical literature.
\end{abstract}

Keywords Mathematical practice $\cdot$ Scientific practice $\cdot$ Applications · Geometry · Voting theory

\section{Mathematical resources and problem-solving}

The last twenty years have seen a remarkable increase in philosophical studies devoted to the role played by mathematics in scientific practice. Work in this area has, at the same time, covered much ground and opened up opportunities for lines of investigation not yet pursued. For instance, the vast amount of work devoted to modelling with differential or integral equations and computer simulations ${ }^{1}$ contrastively points to areas that have not yet received much attention: one of them, to be discussed in the following sections, is structural modelling in mathematical social science. ${ }^{2}$

This area of research is of philosophical interest because its examination can shed much light, in an especially accessible and illuminating way, on specific aspects of the application of mathematics that are recognised to matter but have not been widely studied so far. The aspect of applications I shall be focussing on in this paper has been identified in the following terms by Ardourel, Barberousse and Imbert:

\footnotetext{
1 A small sample of important contributions is Ardourel and Jebeile (2017), Batterman (2006), Bursten (2021), Fillion (2019), Lenhard (2007), Wilson (2006).

2 The single, most extensive discussion of mathematical modelling in social science is the study of econometric and macroeconomic models in Boumans (2005). Here models are typically systems of equations, rather than the structured objects discussed in this paper.
}

$凶$ Davide Rizza

d.rizza@uea.ac.uk

1 School of Politics, Philosophy, Language and Communication Studies, University of East Anglia, Norwich, UK 
When analyzing scientific representations, philosophers of science are keen on mentioning that some models provide scientists with "mathematical resources" and "inferential power", but they seldom give a detailed analysis of these notions. (Ardourel et al. 2018, p. 2)

The authors of Ardourel et al. (2018) provide part of the missing analysis for a specific family of resources, namely mathematical formalisms. They also note that structures and the mathematical results associated with them constitute natural mathematical resources but do not devote their investigation to them. It is the goal of this paper to do so through a study of significant episodes in mathematical social science, more specifically mathematical voting theory.

In order to understand the role of structural resources within scientific practice, it seems to me especially helpful to look at them in connection with problem-solving. Something functions as a resource if it can support a particular activity. Thus, being a resource is best spelled out in connection with a problem-solving activity. Structures or structured objects play the role of resources if they help tackle problems within a field of enquiry. When that happens, the generic qualification of "inferential power" associated with their employment can be more informatively reduced to the effectiveness of the methods erected upon them.

In order to provide a sufficiently thorough philosophical elaboration of the last remarks, it is helpful to identify certain turning points within scientific problemsolving, in which structural objects play an important role. I refer to these stages of enquiry simply as phase (a), phase (b) and phase (c). In outline, phase (a) is a stage at which the terms of certain problems under investigation are assigned formal characters in such a way that the problems themselves can be referred to a formal working environment. Phase (b) is a stage at which the given environment is used to introduce problem-solving techniques. Phase (c) is the stage at which the formal working environment from phase (a) is modified to assimilate new problems and the store of techniques from phase (b) is expanded to deal with such new problems. I shall refer to phases (a) to (c) to articulate the analysis of concrete problem-solving episodes in scientific practice. A further clarification of what these phases amount to can only be offered through the examination of relevant enquiries, which occupies the rest of the paper.

In particular, Sect. 2 provides a more detailed account of phases (a) to (c) in terms of a quick, preliminary illustration. Sections 3 and 4 , on the other hand, offer a progressively broader account of these phases in the context of more extensive problem-solving activities.

In the remainder of the present section I only wish to show how (a) to (c) can be rather naturally singled out in a contrastive manner, by looking at aspects of the application of mathematics that have been neglected by recent, influential lines of investigation.

1. Over the last few decades, many contributions to philosophy of mathematics have portrayed applications in terms of a correspondence between an empirical and a mathematical structure. Two frequently cited sources are Field (1980), p. 6 and 
Bueno and Colyvan (2011), pp. 346-347 3 ). The latter article recognises that the world does not come equipped with a set of objects [...] and sets of relations on those' (Bueno and Colyvan 2011, p. 347), i.e. as a cluster of structured settings. Nonetheless, the problem of examining how structured settings are arrived at is not raised or pursued. The decision to neglect this issue may be due to a remark on $\mathrm{p}$. 354 , to the effect that empirical settings are not required to be given a mathematicsfree description. The remark is not unreasonable ${ }^{4}$ but it shifts attention away from the fact that it is an important phase of scientific investigations to introduce a structured presentation of relevant contents that supports the progress of enquiry. The introduction of such a presentation is phase (a).

2. The stress of Bueno and Colyvan (2011) on the world in the quote from 1 above is, in my opinion, more fruitfully replaced by a stress on problems. The fact that scientific enquiry is a human activity endows it with a distinctive epistemological status. It is a confrontation between an agent and her surroundings in which means have to be devised in order to make those surroundings decipherable and to enable specific interventions upon them. What is pressing, under such circumstances, is the resolution of problems. Desired information is to be gained and specific goals (e.g. predictive or technological) are to be achieved. Thus, if phase (a) is to support the progress of enquiry, it must do so by introducing a structured environment in which it is possible to develop problem-solving techniques. Their construction is carried out in phase (b).

3. An independent motivation for phase (b), as well as a motivation for phase (c), may be spelled out by considering a recent topic of dominant philosophical interest in connection with the application of mathematics, i.e. mathematical explanation. Contributions to the debates on mathematical explanation look at scenarios in which mathematical resources are already available to account for certain given facts. Such scenarios, especially as they are connected to the problems of clarifying whether or not a mathematical result has explanatory power, exclude an interest in the work done before the resources used to provide explanations were available. This work is the construction of problem-solving techniques, i.e. phase (b). Moreover, work on mathematical explanation (as important articles on this topic like Lyon (2012), Lange (2013), Lange (2017) show) has typically restricted attention to the use of individual mathematical results to explain individual phenomena. Specialised attention to contexts of the latter kind naturally leads to a neglect of broader ones, in which not only many interrelated problems are initially present, but systematic attempts to relate them to new problems is made. When these attempts are made the subject of specific analysis, phase (c) emerges.

It is worth clarifying that my goal, when making the previous points, was not to criticise existing approaches, with a view to, say, rejecting them, but simply to rely upon them to identify neglected aspects of applications. In this connection, I do not deny that many current, wide-ranging studies of applications are sensitive to the aspects

\footnotetext{
3 It may be noted that the views expressed in these contributions revive, in effect, some elements of an account of mathematical modelling offered in Coombs et al. (1954).

4 Although, in the absence of any limits to the mathematical description of an empirical setup, applications of mathematics can proceed without the immersion and interpretation steps prescribed by the inferential model advocated in Bueno and Colyvan (2011).
} 
of applications I am drawing attention to. A dedicated study of precisely these aspects is, however, still missing and my aim is to supply it (or to begin to supply it).

For instance, the interest in classifying the epistemic contributions of mathematics to scientific enquiry, which drives extensive analyses in Pincock (2012), falls in line with the aims of this paper. The epistemic contributions I focus on are not, however, part of Pincock's discussion, largely because of its emphasis on representation, as opposed to problem-solving.

More generally, I am hoping to provide, in what follows, a study of applications from a viewpoint that can constructively enrich several ongoing lines of investigation by exploring areas that they have been neglected (as in the case of Bueno and Colyvan (2011) or Pincock (2012)) or by extending their purview beyond issues they have already applied themselves to (as in the case of Ardourel et al. (2018)).

\section{Phases of problem-solving}

My goal in this section is to offer a more concrete impression of what phases (a) to (c) are, before studying them in greater detail and within broader contexts. It is important to recall that, in general, phases (a) to (c) emerge because problems that currently resist solution have arisen. Phase (b) introduces mathematical techniques designed to tackle them. Their introduction is, in structural modelling, made possible by the assignment of formal characters to the terms of the given problems. Only if these problems' terms can be regarded in reasoning as particular mathematical items can mathematical techniques be devised to act upon them. This is why problems have to be set within a suitable mathematical environment before they can be tackled. Phase (a) sets them in the environment that supports phase (b).

In general, the execution of phases (a) and (b) does not merely lead to the resolution of pre-existing problems. It also produces a richer conception of these problems, refining existing ones and relating them to new problems. The environment originally set up in phase (a) may prove inadequate to deal with some new problems: when this is the case, phase (c) takes place. The original environment is modified through the addition of new features or the integration into a more encompassing environment, from which new problem-solving techniques emerge.

As a miniature illustration of phases (a), (b) and (c), I now wish to consider a simple practical problem. The problem is to identify published books by numerical codewords for the purpose of ordering, sales reporting and inventory control. Useful codewords enable the detection of errors. For instance, it should be possible to tell if a printing error swapped two digits of a codeword. ${ }^{5}$ This is a highly circumscribed problem belonging to the vastly larger domain of coding theory.

Fixing the length of a codeword at ten digits, it is useful to let the numerical digits denote the elements of a structured object, the formal working environment. An obvious choice for the set of codeword digits is $\{0,1, \ldots 9\}$, but, in order to supply an environment on which addition and multiplication are well-behaved, it is better to

\footnotetext{
5 Error-detection dispenses with checks against an authentic copy of the codeword.
} 
adopt $\{0,1, \ldots, 10\}$. The latter set, which I shall refer to as $Z_{11}$, can be endowed with canonical field structure. ${ }^{6}$

Phase (a) amounts to the introduction of $Z_{11}$ for the purpose of carrying out phase (b), i.e. the design of an error-detecting code. ${ }^{7}$ One possibility is to let codewords be of the form $x_{1}, \ldots, x_{10}$, where the first nine digits $x_{1}, \ldots, x_{9}$ identify a book, its publisher and its language, as desired, while $x_{10}$, the tenth digit, ${ }^{8}$ satisfies the following equality modulo 11:

$$
\sum_{i=1}^{10} i x_{i}=110
$$

The above modular equality entails that its sides are both multiples of 11 . Field arithmetic on the environment $Z_{11}$ now guarantees the sought error-detecting feature. Swapping two digits in a codeword produces a string that is not a codeword (does not satisfy the last equality). To see how, let a swap of $x_{m}, x_{n}$ in the codeword $x_{1}, \ldots, x_{10}$ yield the word $y_{1}, \ldots, y_{10}$. Then:

$$
\sum_{i=0}^{10} i y_{i}=11 \sum_{i=0}^{10} i x_{i}+(n-m) x_{m}+(m-n) x_{n}={ }_{11}(n-m)\left(x_{m}-x_{n}\right) \neq_{11} 0 .
$$

and $y_{1}, \ldots, y_{10}$ is not a codeword because, in $Z_{11}$, the product of two nonzero elements must be nonzero. For the same reason, single incorrect digits are detected. Thus, the algebraic structure of the environment $Z_{11}$ enables error-detection features and helps set up a code that solves the given problem. ${ }^{9}$ The construction of a code, obtained by defining codewords in terms of a relation on $Z_{11}$ is phase (b). Longer codes with a prime number of digits and an error-detecting features can be constructed along similar lines, using prime fields other than $Z_{11}$.

The simple code construction described here highlights the usefulness of finite fields as formal working environments. Phase (c) ensues when other coding problems are taken into account. For instance, problems in cryptography require the introduction of linear codes, studied within the formal working environment of vector spaces over finite fields.

The quick sketch just offered can help paint a more concrete picture of phases (a) to (c), but only in a very limited form. The environment in phase (a) is not always a structure in the model-theoretic sense. Phase (b) does not always reduce to the solution of a single problem: in general, its goal is to tackle, refine and extend a family of initially given problems. Phase (c) is not always a familiar integration of structures but may require finding manageable ways of enriching an environment or amalgamating distinct environments.

\footnotetext{
6 More explicitly, with addition and multiplication modulo 11. Sums and products are computed by taking the remainders of division by 11 of the usual arithmetical sums and products. Multiplication modulo 10 rules out field structure because it allows zero divisors, e.g. $2 \cdot 5=0$ modulo 10 .

7 A code is just a set of codewords. Obviously, useful codes have distinctive features.

8 If the value of $x_{10}$ required by the condition below is 10 , the symbol $X$ is used.

9 It may be worth noting that was just described is the familiar ISBN code.
} 
The additional dimensions of phases (a) to (c) that could not be taken into account here are explored in the next two sections, which consider progressively more extensive contexts of enquiry within mathematical voting theory. This research area is of special interest because important advances in it have arisen from the construction of geometric working environments. These environments, in turn, have supported the introduction of ingenuous problem-solving techniques.

\section{A simple geometric environment}

Voting, a collective decision method, relies upon a procedure to associate inputs from voters with an outcome, which may be the choice of an action, a candidate or a policy. It has long been known that voting procedures give rise to undesired or puzzling results. A famous example, originally described by Condorcet in Condorcet (1785), pp. xvi-xviii, involves nine voters and three candidates $A, B, C$. Four voters express the ranking $A>B>C$ (where the inequality symbol denotes strict subjective preference), three express the ranking $B>C>A$ and two express the ranking $C>A>B$.

The rule of pairwise majority, combining together the outcomes of three subelections on the respective pairs $(A, B),(B, C)$ and $(C, A)$, produces the cyclic outcome $A>B, B>C, C>A$, from which it is impossible to select a winner. The study of cyclic outcomes has been a major theme in modern voting theory since at least Arrow's theorem (see Arrow 1951). Condorcet's approach, which focusses on a special procedure (i.e. pairwise majority), has been replaced and generalised by the study of conditions that force any procedure satisfying them to produce Condorcet-type situations, in which certain transitive rankings yield a cyclic outcome.

In this context, a voting procedure is usually represented in set-theoretic terms. Candidates or alternatives are regarded as a finite, nonempty set $S$ (with at least three elements). Given $n$ voters, if $L(S)$ is the set of linear orders on $S$, an element of $L(S)^{n}$ is a list of $n$ strict preferences or a preference profile and a voting procedure may be abstractly regarded as a function $f: L(S)^{n} \longrightarrow L(S)$. The problematic occurrence of cyclic outcomes is often translated into the statement of conditions that cannot be satisfied by any function $f$ of the type just described.

Investigations into the unsatisfiability of conditions that force cyclic outcomes is of interest here because it supplies a formal context for the position of problems that cannot be satisfactorily tackled without the introduction of a suitable working environment. This is apparent from a classic contribution to social choice theory (i.e. Sen 1970), due to Amartya Sen. Sen observed that the following conditions cannot be simultaneously satisfied by any function from $L(S)^{n}$ to $L(S)$ :

1. at least two voters are decisive on two respective, distinct pairs of alternatives (i.e. the way they rank those pairs is the way they are ranked by the voting procedure);

2. if every voter exhibits the same ranking of a given pair of alternatives, this is the procedure's ranking;

Conditions 1 and 2, known as Minimal Liberalism and the Pareto condition respectively, always force some cyclic outcome. To establish his result, Sen followed Condorcet's approach and described a simple, two-voter scenario in which one voter, 
decisive on the pair ( $A, B)$, expresses the ranking $C>A>B$, while a second voter, decisive on the pair $(B, C)$, expresses the ranking $B>C>A$. The pairwise outcome of the profile just described is a cycle.

Sen's cyclic scenario raises one interesting issue. Its cyclic outcome depends only on the pairwise rankings $C>A, A>B$ from one voter and only on the pairwise rankings $C>A, B>C$ from the other voter. These rankings can be regarded indifferently as sub-rankings of transitive or of cyclic rankings. Thus, Sen's specific example does not make use of the assumption that a procedure should take profiles of transitive rankings as arguments. The problem of determining whether this is always the case when cyclic outcomes occur naturally arises.

This problem is important because a solution shows whether or not Sen's conditions are sensitive to the transitive behaviour of voters. The desired solution requires a comprehensive understanding of the manner in which Sen's conditions 1 and 2 operate. Specific cyclic examples do not provide a comprehensive picture. All possible cyclic outcomes forced by conditions 1 and 2 must be taken into account in order to determine whether each one of them can be supported by transitive rankings. A priori, it cannot be ruled out that there may be cyclic outcomes not supported by transitive rankings.

A general analysis of the cycles determined by conditions 1 and 2 requires combinatorial considerations: it essentially concerns the way pairwise rankings of alternatives in a profile are connected to outcomes under the action of conditions 1 and 2. The combinatorial connection is not apparent. An environment is needed to organise the arbitrary combinations of pairwise rankings into a structured object that supports techniques of analysis. The needed organisation is achieved in phase (a).

For the sake of clarity, I illustrate phase (a), as carried out in Li and Saari (2008), only with respect to three alternatives $A, B, C$ (the same approach generalises to any finite number of alternatives). The formal working environment is set up through the assignment of two endpoints on a unit segment to the two strict rankings of $(A, B)$. The strict rankings of $(B, C),(C, A)$ are similarly associated with endpoints of other unit segments. When these segments are located on the axes of an orthogonal system, as in Fig. 1, the unit cube emerges as a structured object supporting the analysis of conditions 1 and 2 . The cube's vertices carry ranking information: for instance, vertex 8 from Fig. 1 codifies the pairwise rankings $B>A, A>C, C>B$. Moving away from the origin along one axis reverses exactly one of these rankings. Note that vertices 7, 8 codify cycles and that each edge emanating from a cycle is incident on a transitive vertex (a vertex codifying a transitive ranking).

The formal working environment just instituted supports phase (b) for two reasons: (i) it allows a simultaneous geometric representation of profiles and outcomes; (ii) because of (i), it provides a technique to study pairwise comparisons by working with the incidence geometry of the unit cube (hypercubes support similar analyses for more than three alternatives ${ }^{10}$ and may be regarded as an expansion of the formal working environment just described).

Because of (i) and (ii), a geometric technique can be developed to study all cyclic outcomes. This technique relies on the simple fact that conditions 1 and 2 are, in the geometric environment, ways of selecting cube faces. For instance, whenever the

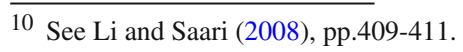


Fig. 1 Pairwise comparison environment

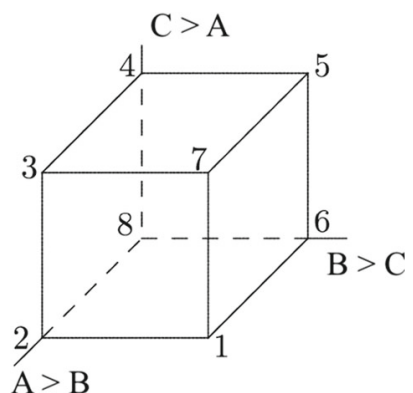

$\mathrm{A}>\mathrm{B}$

Pareto condition applies, the rankings of every agent must lie on the same face (e.g. the pairwise outcome $A>C$ requires the bottom face in Fig. 1). If, in addition, an agent is decisive, her ranking is restricted to a specified edge on that face. No further restrictions ensue, because no agent is decisive on more than one pair.

Thus, profiles supporting a given outcome (a vertex) lie on certain edges of a cube's face. It follows that every cycle is supported by transitive profiles. This is because the cyclic vertices 7, 8 are linked to transitive vertices in each direction. To see why this matters, consider the cyclic outcome 8 . The Pareto condition can be invoked to select the cube's bottom face, on which 8 lies. The rankings of decisive agents must be taken on the edges emanating from 8 , so they are the transitive rankings of vertices 2 and 6 . Finally, the transitive ranking of any other agent can be chosen from the transitive vertices on the bottom face of the cube (i.e. 1, 2, 6).

The last considerations yield a uniform method to build all examples of cycles produced by Sen's conditions. It suffices to select a cyclic vertex and consider the profile in which every voter expresses the same cycle: condition 1 allows moves of voters preferences along edges incident on the cyclic vertex, thus producing the same cyclic outcome on the basis of transitive rankings.

It is worth noting that the technique just described works beyond the threedimensional case, for a simple reason. A decision outcome lies on the intersection of all faces selected by conditions 1 and 2, whereas an individual ranking lies on the intersection of some faces, which includes the intersection of all faces. Thus, a cyclic outcome is always supported by unanimous cyclic rankings and these rankings can always be turned into transitive ones with the same outcome, using condition 1 to visit adjacent edges or faces (allowing for the added freedom supplied by hypercubes).

Sen's conditions alone do not provide information on whether there are cyclic outcomes supported only by transitive profiles or cyclic outcomes that are never supported by unanimously cyclic profiles ${ }^{11}$. The geometric technique developed in phase (b) makes use of the unit cube to obtain the missing information. Moreover, because this technique operates on disconnected pairwise rankings, using only the incidence geometry of the unit cube, it never relies on the transitivity of voters' rankings. It follows that Sen's conditions 1 and 2 operate uniformly irrespective of whether or not voter behaviour satisfies transitivity.

11 In fact, the literature on Sen's theorem predating Li and Saari (2008) has often had to struggle with fairly intricate constructions of cycles from transitive information (see e.g. Gibbard (1974) and Gaertner et al. (1991)). 
It should now be clear that phase (b) provides a way of engaging with conditions 1 and 2 that supports systematic analyses (e.g. by providing a uniform method to generate cyclic outcomes from transitive profiles) and solves the problem posed by the fact that Sen's example disregards the transitivity of individual rankings. The disregard is not accidental: it accompanies every cyclic outcome constrained by conditions 1 and 2 .

The analysis supported by the geometric technique from phase (b) can be further refined by one form of phase (c), namely the addition of structural features to the geometric environment. A simple but insightful instance of phase (c) depends on the addition of topological structure to the faces of the cube, now regarded as a subset of $\mathbb{R}^{3}$, as opposed to a point lattice.

The idea is to focus on a specific face $F$ (singled out by the Pareto condition) and take the points of $F$ to codify the ranking of the vertex closest to them or a tie (in case they lie on the midpoint of an edge or on a line joining two midpoints and parallel to an edge). The notion of closeness now in place ${ }^{12}$ can be used to deduce that any pairwise outcome determined by conditions 1 and 2 is close to a particular average of the supporting profiles ${ }^{13}$. Thus, a cyclic outcome of transitive rankings, e.g. 8 , reflects a preponderance of hypothetical cyclic contributions because it is close to an average of mostly non-transitive profiles ${ }^{14}$. It is possible to conclude, again without making any appeal to the transitivity of voters' rankings, that, whenever a procedure satisfying conditions 1 and 2 produces a cycle, it

essentially ignores the specified profile with transitive preferences; instead, it reports the averages of all supporting profiles where most are cyclic (Li and Saari 2008, p. 404).

Phases (a) to (c), in the form just described, show that being in possession of a formal approach is not the same thing as being able to control the interrelated problems that may emerge from it. The formal context that yields Sen's result raises at the same time problems that only a focussed analysis of the constraints set by conditions 1 and 2 on the combinatorial space of pairwise rankings can solve. The analysis is not supported by the formal elements of Sen's formulation but requires a new environment and a new associated technique. Their employment issues into a refined analysis, which is sharpened by the structural enrichment effected in phase (c).

The outcome of the geometric approach described in this section is not only a better grasp of the initial formal setting (the main goal of problem-solving in this context) but also an expansion of the types of theoretical engagements possible with Sen's result. For instance, supporting profiles for a given outcome can now be systematically listed, transitive profiles that support cyclic outcomes can be constructed by a uniform method and higher-dimensional variations of Sen's original example can be searched for and exhibited.

In general, phases (a) to (c) do not multiply the engagements with a given problem only in a single direction (all attention so far has been paid to cyclic outcomes) but in

\footnotetext{
12 For a more formal definition of 'closeness', see the Appendix.

13 See the Appendix or Li and Saari (2008), p.411 for details.

14 With two voters, three out of the four supporting profiles $(8,8),(2,6),(2,8),(8,6)$ contain cyclic rankings.
} 
multiple directions. They also lead to multiple refinements of initially given problems. The next section is devoted to examining these phenomena in the context of voting problems different from those encountered so far.

\section{Geometry of voting}

Condorcet's work shows that voting problems arise relatively easily from direct experimentation with hypothetical scenarios. The following one, adapted from Saari (2008), p. 2, highlights issues that require a more extensive mobilisation of structural resources than the problems considered in the preceding sections. Suppose that a company's executive committee, composed of fifteen members, must choose one of the following investment policies: (A) invest all reserves into financial derivatives; (B) invest half of the reserves into financial derivatives; (C) invest none of the reserves into financial derivatives. Once preferences are declared, let the result be:

6 committee members prefer A to B to $\mathrm{C}$;

5 committee members prefer $\mathrm{C}$ to $\mathrm{B}$ to $\mathrm{A}$;

4 committee members prefer $\mathrm{B}$ to $\mathrm{C}$ to $\mathrm{A}$.

Since there is no unique way of aggregating preferences, a voting procedure must be chosen for an outcome to emerge. A widely used procedure, known as plurality, counts top-ranked alternatives. Under plurality, the outcome is:

$$
A>C>B,
$$

and the company's reserves are invested into financial products. Note that, despite this outcome, most committee members consider $A$ to be the least desirable alternative. A different voting procedure could prevent the selection of $A$. If, for instance, pairwise majority was adopted, the outcome would be:

$$
B>C>A,
$$

i.e. the reversal of the plurality outcome. Reflection on this example (and practice with many others) leads to several questions. It is, for instance, reasonable to ask whether, by a judicious choice of procedure, it might not be possible to obtain any prescribed outcome from the same preference profile. The problem implicitly posed consists in determining how many different outcomes can be supported by the same profile, as the voting procedure varies.

A converse problem arises when a procedure is fixed and the profiles vary. The problem is now to determine the degree of variation across outcomes allowed by a fixed procedure, when all possible preference profiles are taken into account. A related problem is whether, given two distinct decision procedures, it is possible to determine outcomes, if any, that one realises and the other does not.

Finally, because the committee scenario highlights a lack of coordination between plurality and pairwise majority, the problem arises of determining whether such lack of coordination is accidental or systematic and whether it vanishes or persists in presence 
of other procedures (e.g. if plurality is replaced by a finer-grained rule taking secondbest options into consideration).

The problems just listed are suggested by a revealing example: they do not, in themselves, deliver an explicit indication of what techniques might provide solutions. An effective response to this state of affairs consists in activities poignantly characterised by the following quote from Pólya's work on mathematical problem-solving. The relevant activities are:

Advancing mobilization and organization of our knowledge, evolution of our conception of the problem, increasing prevision of the steps which will constitute the final argument (Pólya 1990, p.159).

It is easy to regard the above quote as a concise characterisation of phase (a) and phase (b). To mobilise and organise knowledge is to take up the terms of the given problems and regard them symbolically in such a way that they can be integrated into a formal working environment. The environment itself displays an evolved conception of the problems, for three reasons: (i) because the originally given problems are structured within the environment; (ii) because structuring is not portraying abstractly but preparing for use or repurposing for novel theoretical and practical engagements; (iii) because the original problems are refined and new, related problems can be posed and tackled. Finally, the techniques established in phase (b), insofar as they crystallise problem-solving procedures along specific lines, make problem-solving action consequential and, insofar, increase prevision of steps in the sense of Pólya. Phase (c) then appears as a further evolution of a problem's conception or as an evolved conception of distinct problems, which it brings together and subjects to an expanded range of techniques.

These last observations are concretely spelled out in the remainder of the present section, by looking at the way in which the voting problems just posed may be framed and attacked within a geometric environment.

\subsection{Phase (a)}

The problems stated at the beginning of this section concern the action of a voting procedure on profiles under a specified voting rule or a family of voting rules. It is therefore helpful to think of a procedure as a function that links profiles to outcomes under a voting rule. This observation does not provide a formal working environment but identifies its constituents, namely profiles, voting rules, outcomes and procedures. It is plausible to try and construct a formal working environment by linking them together once they have been individually structured: procedures can provide the desired link.

The first step of phase (a) consists in mobilising knowledge (in Pólya's sense) about the items to be integrated into the environment, in order to find a useful way of presenting them as structured objects. Fixing attention on three alternatives ${ }^{15}$, a basic but useful remark is that they determine six possible strict rankings. Any voter can strictly rank the alternatives in only one of six ways. This is to say that information about the

\footnotetext{
15 The methods described below can be adapted to any finite number of alternatives, but they are especially transparent when restricted to three.
} 
Fig. 2 The space $C(3)$

partitioned into ranking regions

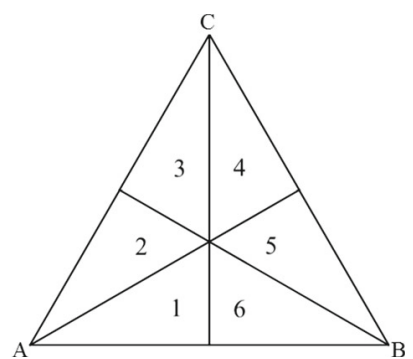

voters' contribution to an election can be summarised by listing the proportions of voters expressing each of the six possible strict rankings.

If the relevant proportions are $p_{1}, \ldots, p_{6}$, any profile is codified by a linear combination of the form:

$$
p_{1}(1,0,0,0,0,0)+\cdots+p_{6}(0,0,0,0,0,1)
$$

where $p_{1}+\cdots+p_{6}=1$ and $0 \leq p_{i} \leq 1$ for $i=1, \ldots, 6^{16}$. This is enough to structure the space of profiles as a convex figure in the Euclidean space $\mathbb{R}^{6}$. This figure is generated by suitable combinations of six vertices. In technical terms, it is the convex hull $C(6)$ of the six unanimity profiles $(1,0,0,0,0,0), \ldots,(0,0,0,0,0,1)$.

Essentially the same line of thinking codifies the space of outcomes as $C(3)$, the convex hull of the three unanimity outcomes $(1,0,0),(0,1,0),(0,0,1)$. More concretely, $C(3)$ is a triangular surface in $\mathbb{R}^{3}$, which is represented as the triangle $\mathrm{ABC}$ in Fig. 2 below. It is important to note that $\mathrm{ABC}$ is partitioned into thirteen equivalence classes, each describing the distinct realisations of the same ranking outcome, with ties included ${ }^{17}$. In particular, each of the six interior regions marked by the numerals 1 to 6 in Fig. 2 contains the outcomes determining one of the six possible strict rankings of $A, B, C$.

An election procedure may now be regarded as a function that makes use of a voting rule to map $C(6)$ into $C(3)$. Not all voting rules may be codified by a convex figure but, fortunately, an entire family of them can.

By definition, a positional rule for three alternatives assigns them scores $s_{1} \geq$ $s_{2} \geq s_{3}$ in a non-constant fashion. Its outcome (a transitive ranking) is unaffected

\footnotetext{
16 In the remainder of the paper, the following assignment of indices to voters' rankings is adopted: 1 labels $A>B>C ; 2$ labels $A>C>B ; 3$ labels $C>A>B ; 4$ labels $B>A>C ; 5$ labels $B>C>A ; 6$ labels $C>B>A$.

17 To see this, consider the midpoint $(1 / 2,1 / 2,0)$ of $A B$, marked in Fig. 2: clearly, it identifies the ranking $A \sim B \succ C$, where $A, B$ are tied and $C$ receives no votes. The points on $A B$ to the left of the midpoint, with the exception of $A$, assign a greater weight to $A$ than they do to $B$ and zero weight only to $C$, so they all express $A \succ B \succ C$. The same ranking is expressed by every point in the interior region marked by the label 1 in Fig. 2. An entirely symmetrical situation (interchanging the roles of $A, B$ ) arises to the right of the $A B$-midpoint and in the interior region 6. The other labeled regions can be treated in a similar manner (for instance, region 2 expresses the ranking outcome $A \succ C \succ B$ ). The barycentre $\mathcal{I}=(1 / 3,1 / 3,1 / 3)$ of $A B C$ (the intersection of its medians) expresses the complete tie and the points along each median express exactly one tie between two alternatives. The six interior regions (with the respective open boundaries), the six semi-open segments (i.e. minus the endpoint $\mathcal{I}$ ) in which the medians cut each other and the point $\mathcal{I}$ are the 13 ranking regions into which $C(3)$ is partitioned.
} 
if a positive, linear transformation is applied to the scores. By this remark, every positional rule is identifiable with a normalised equivalent assigning scores $1-s, s, 0$ to the alternatives, where $s \in[0,1 / 2]^{18}$. Positional rules are thus codified by vectors of the form $(1-s, s, 0)$. When $s=0$, plurality rule arises. When $s=1 / 2$, a voting rule known as antiplurality does. In other words, by a useful quotient, positional rules can be reduced to a segment in $\mathbb{R}^{3}$, namely the convex hull $C(2)$ of plurality and antiplurality.

It is worth noting that the convex figures introduced so far are the constituents of a formal working environment for positional elections, even if they are not structures in the model-theoretic sense ${ }^{19}$. Even though $C(6), C(3), C(2)$ could be immersed in the same ambient space, they function as a working environment on their own. In fact, it is an advantage to consider them in isolation from ambient space, for the sake of working with lower-dimensional objects.

Phase (a) is completed by letting voting procedures link together $C(2), C(6)$ and $C(3)$. The required link is established by the given geometrical data. To see why, let $\mathbf{w}=(1-s, s, 0)$ be a fixed positional vector and $\mathbf{p}=\left(p_{1}, \ldots, p_{6}\right) \in C(6)$ be a fixed profile. Each $p_{i}$ ranks the same three candidates in one of six ways: thus $p_{i}$ times a suitable permutation of the entries in $(1-s, s, 0)$ yields the contribution of voters of type $i$ to a positional outcome. The outcome itself is just the sum of six contributions. Calling $\mathbf{w}^{1}, \ldots, \mathbf{w}^{6}$ the permutations of $\mathbf{w}$ 's entries corresponding to $p_{1}, \ldots, p_{6}$, a plurality procedure based on the rule $\mathbf{w}$ and acting on profile $\mathbf{p}$ can be explicitly codified as the following function:

$$
f(\mathbf{w}, \mathbf{p})=p_{1} \mathbf{w}^{1}+\cdots+p_{6} \mathbf{w}^{6} .
$$

The link between the distinct components of the formal working environment supplied by $f$ plays a central technical role. Because $f$ is linear in both $\mathbf{w}$ and $\mathbf{p}$, it sends convex figures like $C(2)$ and $C(6)$ into convex figures included in $C(3)$. Since, moreover, the convex figures of interest are finitely generated, they can be reconstructed as convex hulls of finitely many $f$-values. The binding role played by $f$ within the formal working environment opens the way to phase (b), which the next subsection examines in detail.

\subsection{Phase (b)}

Several related problem-solving techniques arise from the fact that $f$-images of convex figures are convex.

One of them, which is worthy of close examination, enables the systematic study of positional outcomes on a fixed profile. As noted earlier, the vectors $\mathbf{w}_{0}=(1,0,0)$ and $\mathbf{w}_{1 / 2}=(1 / 2,1 / 2,0)$ generate the segment $C(2)$. For a fixed profile $\mathbf{p}$, the image of $C(2)$ under $f$ is the convex hull of the plurality outcome $f\left(\mathbf{w}_{0}, \mathbf{p}\right)$ and the antiplurality

\footnotetext{
18 For the argument leading to this representation, see Saari (1994), pp. 47-48.

19 The ambient space for the convex figures that have been singled out is endowed at least with linear structure. Then, neither of $C(6), C(3), C(2)$ is a substructures of the space. None of them contains the null vector or is closed under multiplication by a scalar.
} 
outcome $f\left(\mathbf{w}_{1 / 2}, \mathbf{p}\right)$. More concretely, the $f$-image of $C(2)$ is the segment on the triangle $\mathrm{ABC}$ that joins these two outcomes. In other words, the $f$-image of $C(2)$ is the set of all positional outcomes for a fixed profile $\mathbf{p}$ and can be used to study the variation of election outcomes under a change of positional procedure.

More precisely, the study of plurality outcomes rests on both the analytic (coordinate-dependent) and synthetic (coordinate-free) features of the environment. To see this, consider one of the problems raised at the beginning of the section, namely the problem of determining whether an election outcome could be changed at will by suitable changes of procedure. In general, the answer is negative: profiles that allow exactly one positional outcome can be computed. For instance, given the outcome $A>B>C$ in region 1 from Fig. 2 , the profile $(1 / 2,0,1 / 8,3 / 8,0,0)$ can only have that outcome under every positional rule. The geometric fact guaranteeing the latter conclusion is that the $f$-values of plurality and antiplurality are incident on region 1 : since the region is convex, the segment joining the two $f$-values must be included in it (similar considerations hold for any other prescribed outcome).

It is important to realise that the technique enabled by the linearity of $f$ in the argument $\mathbf{w}$ does not simply solve an originally given problems, but also refines it and relates to it new and subtler problems.

An immediate refinement of the given problem consists in determining the largest number of distinct plurality outcomes that any profile will allow. Thirteen distinct outcomes are possible on $\mathrm{ABC}$, but convexity considerations suggest that not all of them can be realised by any one profile, since the positional outcomes must all be collinear.

The interaction between the linearity of $f$ and the betweenness geometry of ABC provides more specific information. Let $\ell$ be the segment joining the plurality and antiplurality outcome. If its endpoints lie on labeled, interior regions of $C$ (3) (see Fig. 2 ), these regions can be the same or distinct. If distinct, they are either adjacent or separated by one or two regions (either counting clockwise or counterclockwise). The last case is the less straightforward one. If, in this case, $\ell$ intersects $\mathcal{I}$ (see $\mathrm{fn} .17$ ), exactly three outcomes occur. Otherwise, there is a line parallel to $\ell$ and intersecting $\mathcal{I}$.

The line $\ell$ is wholly contained in one of the two half-planes determined by that parallel line (this step of the argument relies upon Pasch's axiom). Since any such half-plane, being convex, cannot contain more than four interior regions, the maximum number of strict positional outcomes determined by a fixed $\mathbf{p}$ is 4 . Similar considerations show that the maximum number of positional outcomes with at least one tie is 3. Thus, at most 7 different ranking outcomes can arise from the variation of a positional rule on any profile (for further details, see Saari 1994, pp. 116-118).

A new problem connected to the original one consists in determining whether, for any given positional rules $\mathbf{w}_{1}, \mathbf{w}_{2}$ and two ranking regions $r_{1}, r_{2}$ on $\mathrm{ABC}$, there is a profile $\mathbf{p}$ such that $f\left(\mathbf{w}_{1}, \mathbf{p}\right)$ lies on $r_{1}$ and $f\left(\mathbf{w}_{2}, \mathbf{p}\right)$ lies on $r_{2}$. The coarser problem concerning whether or not positional outcomes can in general be changed by a change of rule becomes here the finer problem of determining whether there are profiles especially vulnerable to manipulation.

The affirmative solution to the problem (see Saari 1994. p. 74) is achieved by taking advantage of analytic considerations. It suffices to note that, in view of equation (1), 
$f\left(\mathbf{w}_{1}, \mathbf{p}\right)$ and $f\left(\mathbf{w}_{2}, \mathbf{p}\right)$ determine three linear equations each ${ }^{20}$ in the six unknowns $p_{1}, \ldots, p_{6}$.

Because the three components of an outcome sum up to 1, when two are known the third is uniquely determined. By this observation, $f\left(\mathbf{w}_{1}, \mathbf{p}\right), f\left(\mathbf{w}_{2}, \mathbf{p}\right)$ are unambiguously identified by four, instead of six, linear equations. Once the linear equation $p_{1}+\cdots+p_{6}=1$, which requires that the unknowns determine a profile, is added, the problem of finding profiles vulnerable to manipulation can be restated as the problem of solving a linear system in five equations and six unknowns.

Dealing with systems of linear equations makes it possible to ascertain the robustness of vulnerability to manipulation, i.e. to determine whether there are entire linear spaces of vulnerable profiles ${ }^{21}$ or, instead, small profile variations suffice to disrupt manipulation (both cases occur, depending on whether or not outcomes involve ties).

This simple instance of problem-solving supported by the convex environments for positional procedures is touched upon to draw attention to the fact that, true to Pólya's quote at the beginning of this section, the conception of the original voting problems has evolved. The increase in problem-solving resources does not coincide merely with an increase in solutions to pre-existing problems, but also with an increase in the store of relevant problems and the detail of their formulation.

Before considering the further expansion of problems and techniques that accompanies phase (c), it is worth noting that the environment supplied in phase (a) sustains multiple techniques. We have seen one revolving around the fact that the $f$-image of $C(2)$ is a segment lying on $\mathrm{ABC}$. We can also make use of the fact that the $f$-image of $C(6)$ is a polygon included in ABC. More precisely, for a fixed positional rule w, the $f$-image of $C(6)$ is the convex hull of the vectors:

$$
f(\mathbf{w},(1,0,0,0,0,0)), \ldots, f(\mathbf{w},(0,0,0,0,0,1)) .
$$

Computation of the last six $f$-values for a specified $\mathbf{w}$ shows what positional outcomes that rule realises. If $\mathbf{w}=\mathbf{w}_{0}$, the values in (2) reduce to $(1,0,0),(0,1,0),(0,0,1)$, whose convex hull is the whole of ABC. As $s$ increases from 0 to $1 / 2$, the set of positional outcomes shrinks until, at antiplurality, the values in (2) reduce to $(1 / 2,1 / 2,0),(1 / 2,0,1 / 2)$ and $(0,1 / 2,1 / 2)$, i.e. the intersections between a median and a side of $\mathrm{ABC}$. It follows that antiplurality realises only one fourth of the plurality outcomes. Specific computations make it possible to determine sets of profiles that are not realised under one positional rule but realised under another ${ }^{22}$.

In this case, as well as in the previous illustrations of phase (b), to tackle an initially stated problem is not simply to solve it by bringing an enquiry to a definitive close, but rather to find a way of generating as much information as can be related to the original problem. It can be readily shown that, in general, different voting rules are associated with different families of outcomes. The geometric environment, however,

\footnotetext{
20 Once vector components are taken separately.

${ }^{21}$ Note that, when linear spaces of solutions exist, their elements with rational components are legitimate profiles.

${ }^{22}$ For instance, if $1 / 2<a \leq 1$ and $b=c$, then $(a, b, c)$ is not an anti-plurality outcome but it certainly is a plurality outcome.
} 
does not take this information as conclusive: it takes it as a point of departure for further investigations, concerning, e.g. the proportion by which outcomes are reduced in the transition from a positional rule to another or the definability of sets of outcomes supported by a particular rule but not by another.

Moreover, the geometric environment itself is not only the support of techniques that solve, refine and expand problems, but can itself be taken as a term of a larger problem. In the present context, this happens when the goal is to carry out a comparative analysis of positional and pairwise majority outcomes. The problem of integrating the geometric environment of positional procedures with a distinct environment for pairwise majority leads to phase (c).

\subsection{Phase (c)}

For the sake of clarity and simplicity, it is convenient to discuss phase (c) only relative to majority on a distinguished pair of alternatives $\{A, B\}$ and plurality (recall that $\mathbf{w}_{0}$ denotes the plurality rule), as opposed to arbitrary positional procedures. The methods shortly to be examined could be expanded to cover other pairwise contributions and any positional method.

A geometric environment for $\{A, B\}$-majority is not too difficult to set up. This procedure must act on the space of profiles $C(6)$ and may be described as follows:

$$
f_{A, B}(\mathbf{p})=\sum_{i=1}^{3} p_{i}-\sum_{i=4}^{6} p_{i},
$$

where the first term of the difference computes the proportion of voters ranking $A$ strictly above $B$ and the second the proportion ranking $B$ strictly above $A$. It follows that $f_{A, B}$ takes values in the segment $[-1,1]$ (other pairs of alternatives would be treated in the same way). Note that pairwise majority could have been chosen to take pairs as values, as opposed to differences, in the same way in which e.g. plurality takes triples as values.

The advantage of using a single number as $f_{A, B}(\mathbf{p})$ is that a comparative plurality / majority outcome only lists four, as opposed to five, numerical components. Geometrically speaking, dimension is not increased beyond necessity. When comparative outcomes are represented as ordered 4-tuples, they may be regarded as products of a plurality outcome and a majority outcome. The resulting, comparative outcome space is then the product of the outcome spaces $[-1,1]$ and $C(3)$, namely the prism $\mathbb{P}=[-1,1] \times C(3)$, depicted in Fig. 3 .

The new formal working environment consists of $C(6), \mathbf{w}_{0}$, and two simultaneous decision procedures taking values in $\mathbb{P}$. It is convenient to amalgamate the procedures into a single, comparative procedure $h: C(6) \longrightarrow \mathbb{P}$ such that:

$$
h(\mathbf{p})=\left(f\left(\mathbf{p}, \mathbf{s}_{0}\right), f_{A, B}(\mathbf{p})\right),
$$

The problem of integrating the positional environment into a comparative environment is not yet completely resolved. It is not known whether the newly instituted 
Fig. 3 The prism $\mathbb{P}$. Numerals label the unanimity outcomes under $h$

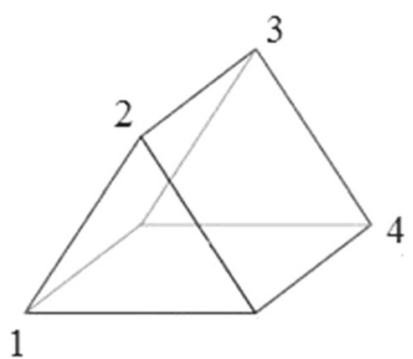

geometric environment sustains problem-solving techniques. A reasonable way to proceed is to see how far the methods developed in phase (b) can be pushed. Convexity considerations continue to apply, since the $h$-image of $C(6)$ is the convex hull generated by the values of $h$ at the unanimity profiles. These $h$-values are labelled in Fig. 3 and determine a tetrahedron ${ }^{23}$. The analysis of positional outcomes, which could be carried out on a simple and well-behaved outcome space (i.e. the equilateral triangle $\mathrm{ABC}$ ), is now replaced by comparative analysis of the same type on a higher-dimensional object.

The increase in dimension produced by the comparative analysis environment is an adversity that motivates the search for a way to transfer the same analysis to a lower-dimensional object. This situation provides, in a simple context, the structural analogue of a predicament familiar from modelling with differential equations, which arises when the existence of an exact but intractable solution prompts the search for manageable approximations. The relevant contrast here is not between exact and approximate but more generally between intractable and tractable. Its significance lies in the fact that intractability requires an adjustment of mathematical methods.

In our context, the problem of finding a setup for lower-dimensional comparative analysis is tackled by the construction of a suitable system of coordinates for comparative outcomes (see Saari 1994, p. 127). The new coordinates are obtained by normalising $p_{i}$ relative to $d=p_{1}+p_{2}+p_{3}$, if $i=1,2,3$, and relative to $1-d$ otherwise. What is being exploited by the normalisations is the fact that voters of type $1,2,3$ (respectively $4,5,6$ ) rank $A$ above $B$ ( $B$ above $A$ ), i.e. they are of the same $\{A, B\}$-majority type.

Any profile $\mathbf{p}=\left(p_{1}, p_{2}, p_{3}, p_{4}, p_{5}, p_{6}\right)$ splits into the profiles

$$
\mathbf{p}_{A B}=\left(p_{1}, p_{2}, p_{3}, 0,0,0\right)+\left(0,0,0, p_{4}, p_{5}, p_{6}\right) \text { and } \mathbf{p}_{B A}=\left(0,0,0, p_{4}, p_{5}, p_{6}\right),
$$

of opposite majority types. It now suffices to set:

$$
\alpha=h\left(\mathbf{p}_{A B}\right)=\left(\left(\frac{p_{1}+p_{2}}{d}, 0, \frac{p_{3}}{d}\right), 1\right) .
$$

The point $\alpha$ lies on the triangular face of the prism $\mathbb{P}$ containing the edge that joins 1 and 2 . In a similar manner, $\mathbf{p}_{B A}$ determines a point $\beta$ on the edge joining 3, 4 .

$\overline{23}$ They are: $1=((1,0,0), 1) ; 2=((0,0,1), 1) ; 3=((0,0,1),-1) ; 4=((0,1,0),-1)$. 
Comparative outcomes can now be described in terms of the majority-type coordinates $\alpha, \beta$. More precisely, they are convex combinations of $\alpha$ and $\beta$, of the form:

$$
h(\mathbf{p})=d \alpha+(1-d) \beta .
$$

The majority-type coordinates deliver a dimensional reduction for a synthetic reason, i.e. because, by Thales theorem, it is possible to project the segment joining $\alpha$ and $\beta$, as well as the outcome $q=d \alpha+(1-d) \beta$, on a triangular face of $\mathbb{P}$, without altering proportions between the distances among $\alpha, q, \beta$.

Comparisons between majority and plurality outcomes can thus be carried out in the lower-dimensional space $C$ (3). The use of $\alpha, \beta$ carries its own problem-solving techniques. Since $\alpha, \beta$ are projected on $A B, A C$ respectively, geometrical considerations show that the midpoint of $\alpha, \beta$ can be placed in any prescribed ranking region of $C$ (3). It now follows that the plurality outcome for $\{A, B\}$ is entirely independent of the corresponding majority outcome ${ }^{24}$. The lack of coordination between plurality and pairwise majority detected by the committee scenario at the beginning of this section is not an accident.

Much like the positional analysis in phase (b), the comparative analysis in phase (c) can be refined and extended. It is, for instance, possible to ask how decisive the victory of $A$ over $B$ must be for a specified positional rule not to reverse the pairwise majority outcome; it may be asked whether or not distinct positional outcomes are also independent of each other; it may be asked whether positional procedures other than plurality are better coordinated with pairwise majority outcomes, and so on. The techniques introduced are able to answer the questions just posed. Their applicability is nothing but their ability to promote new and manifold interactions with given problems.

\section{Concluding remarks}

Philosophers working on scientific and mathematical practice have repeatedly stressed the central role of agents (see e.g. the survey of agent-based studies by Jessica Carter in Carter (2019) and Hasok Chang's wide-ranging discussion of agents in Chang (2011)) and, as a consequence, the philosophical relevance of the activities, purposes and aims that underlie and motivate specialised enquiries.

It seems to me that the dual notion to that of an agent is the notion of a problem. The engagements of agents point to problematic situations and problem-solving techniques. Symmetrically, problems refer to activities and resources mobilised to carry them out and to achieve desired goals. Thus, one may look at agents pursuing specific aims, finding obstacles in their way and seeking to overcome them or, dually, at problems arising in the course of an enquiry, techniques deployed to tackle them, developments and revisions of such techniques.

In a similar way, phases (a) to (c), which were singled out as salient steps in problem-solving, may be regarded as activities that promote novel transactions with familiar circumstances. For instance, the problem of book classification in Sect. 2

\footnotetext{
24 An election in which a strong majority prefers $A$ over $B$ may see $A$ lose to $B$ under the plurality rule.
} 
leads to a reorganised setting in which an error-detecting code is in use. The problem of understanding the impact of decisive agents and unanimity on decision-making leads, in Sect. 3, to designing procedures unaffected by cyclic outcomes (see e.g. Li and Saari 2008, pp. 406-408 and (Petron and Saari 2006, pp. 274-279). Finally, the geometric techniques explored in Sect. 4 lead to new analyses of political elections (an application to presidential elections in the USA may be found in Tabarrok (2001)) and new evaluations of decision procedures.

The expansion of activities is concomitant with the organisation of knowledge. Subject matter that initially appears under the guise of brute facts or puzzling circumstances acquires structural properties as a result of an agent-driven endeavour. The fact, stressed by Dewey, that 'new formal properties accrue to subject matter in virtue of its subjection to certain types of operation' (Dewey 2008, p. 105) is a distinctive feature of scientific enquiry that highlights its formative character.

Again, two complementary perspectives can be taken, depending on whether one wishes to stress the formative effects of an agent's intervention on certain materials or the formative functions associated with the development of problems through phases (a) to (c). In both cases, the materials of experience are structured in the course of a continuous process; at the same time, their newly acquired structural organisation refines and regulates the further conduct of enquiry.

\section{Declarations}

Human or animal rights I confirm that the paper 'Applications in practice' has not been supported by any sources of funding other than the paid research time included in my current academic contract and that, to the best of my knowledge and awareness, no conflicts of interest affect it. I also confirm that the research contained in the paper I have submitted for consideration did not involve any human participants or animals.

Open Access This article is licensed under a Creative Commons Attribution 4.0 International License, which permits use, sharing, adaptation, distribution and reproduction in any medium or format, as long as you give appropriate credit to the original author(s) and the source, provide a link to the Creative Commons licence, and indicate if changes were made. The images or other third party material in this article are included in the article's Creative Commons licence, unless indicated otherwise in a credit line to the material. If material is not included in the article's Creative Commons licence and your intended use is not permitted by statutory regulation or exceeds the permitted use, you will need to obtain permission directly from the copyright holder. To view a copy of this licence, visit http://creativecommons.org/licenses/by/4.0/.

\section{Appendix}

Given two voters and three alternatives, any pairwise outcome determined by conditions 1 and 2 is the average of all averaged supporting profiles.

We focus on the top face $F$ of the unit cube from Fig. 1, selected by the unanimous $C>A$ ranking. The decisive voters impose the $(A, B)$ and $(B, C)$ rankings respectively. Think of $F$ as a region of $\mathbb{R}^{3}$. The midpoints of $F$ 's sides represent ties between alternatives (e.g. the midpoint of the edge joining 2 and 8 represents $A \sim B$ ). Take the basic open sets in $F$ to be open rectangles. If $a \in F$ and $x$ is a vertex of $F, a$ is close to $x$ if, and only if, there is a basic open set $U$ such that its closure contains $a, x$ and no other vertex than $x$. Note that $a$ can be close to more than a single vertex. If 
$a, x$ are as above, $a$ expresses $x$ if, and only if, $a$ is close only to $x$. Points close to exactly one vertex express its ranking. The cycle 7 at vertex $(1,1,1)$, is supported by four distinct profiles. The voter decisive on $A, B$ supports 7 either by the ranking 7 or the ranking 3 , whose coordinates are $(1,1,1)$ and $(1,0,1)$ respectively. The voter decisive on $B, C$ supports 7 either by the ranking 7 or the ranking 5 , whose coordinates are $(1,1,1),(0,1,1)$. If we average each of the four supporting profiles (i.e. take the average of its two components), each resulting average expresses 7 . The average of these points is:

$$
\frac{1}{4}((1,1,1)+(1,0,1)+(1,1,1)+(0,1,1))=\frac{1}{4}(3,3,4)=\left(\frac{3}{4}, \frac{3}{4}, 1\right),
$$

which expresses 7. The argument is similar for vertices other than 7.

\section{References}

Ardourel, V., Barberousse, A., Imbert, C.: Inferential power, formalisms, and scientific models (2018). http://philsci-archive.pitt.edu/15165/

Ardourel, V., \& Jebeile, J. (2017). On the presumed superiority of analytical solutions over numerical methods. European Journal for Philosophy of Science, 7, 201-220.

Arrow, K. (1951). Social choice and individual values. Hoboken: Wiley.

Batterman, R. (2006). On the specialness of special functions (the nonrandom effusions of the divine mathematician). The British Journal for the Philosophy of Science, 58, 263-286.

Boumans, M. (2005). How economicsts model the world into numbers. London: Routledge.

Bueno, O., \& Colyvan, M. (2011). An inferential conception of the application of mathematics. Noûs, 45, $345-374$.

Bursten, J. (2021). The function of boundary conditions in the physical sciences. Philosophy of Science, $88,234-257$.

Carter, J. (2019). Philosophy of mathematical practice - motivations, themes and prospects. Philosophia Mathematica, 27, 1-32.

Chang, H. (2011). The philosophical grammar of scientific practice. International Studies in the Philosophy of Science, 25, 205-221.

Condorcet, J.A.N.d.C.m.d.: Essai sur l'application de l'analyse à la probabilité des décisions rendue à la pluralité des voix. L'Imprimerie Royale (1785)

Coombs, C., Raiffa, H., \& Thrall, R. (1954). Some views on mathematical models and measurement theory. Psychological Review, 61, 132-144.

Dewey, J. (2008). The Later Works of John Dewey, 1925-1953: 1938 - Logic: the Theory of Inquiry, edited by J.A. Boydston. Southern Illinois Press.

Field, H. (1980). Science without numbers. Oxford: Blackwell.

Fillion, N. (2019). Conceptual and computational mathematics. Philosophia Mathematica, 27, 199-218.

Fillion, N., \& Corless, R. M. (2014). On the epistemological analysis of modeling and computational error in the mathematical sciences. Synthese, 191, 537-546.

Gaertner, W., Pattanaik, P., \& Suzumura, K. (1991). Individual rights revisited. Economica, 59, 161-177.

Gibbard, A. (1974). A pareto-consistent libertarian claim. Journal of Economic Theory, 7, 388-410.

Lange, M. (2013). What makes a scientific explanation distinctively mathematical? The British Journal for the Philosophy of Science, 64, 485-511.

Lange, M. (2017). Because without cause: non-causal explanations in science and mathematics. Oxford: Oxford University Press.

Lenhard, J. (2007). Computer simulation: The cooperation between experimenting and modeling. Philosophy of Science, 74, 176-194.

Li, L. I., \& Saari, D. (2008). Sen's theorem: Geometric proof, new interpretations. Social Choice and Welfare, 31, 393-413. 
Lyon, A. (2012). Mathematical explanation of empirical facts, and mathematical realism. Australasian Journal of Philosophy, 90, 559-578.

Petron, A., \& Saari, D. G. (2006). Negative externalities and sen's liberalism theorem. Economic Theory, $28,265-281$.

Pincock, C. (2012). Mathematics and scientific representation. Oxford: Oxford University Press.

Pólya, G. (1990). How to solve it (2nd ed.). New York: Penguin.

Saari, D. (1994). Geometry of Voting. New York: Springer.

Saari, D. (2008). Disposing dictators, demystifying voting paradoxes. Cambridge: Cambridge University Press.

Sen, A. (1970). The impossibility of a paretian liberal. Journal of Political Economy, 78, 152-157.

Tabarrok, A. (2001). President Perot or fundamentals of voting theory illustrated with the 1992 election. Public Choice, 106, 275-297.

Wilson, M. (2006). Wandering Significance. Oxford: Oxford University Press.

Publisher's Note Springer Nature remains neutral with regard to jurisdictional claims in published maps and institutional affiliations. 